\title{
Understanding quantum wires with circular bends
}

\author{
D. W. L. Sprung and Hua Wu \\ Department of Physics and Astronomy, McMaster University, Hamilton. Ontario L8S 4M1, Canada
}

\begin{abstract}
J. Martorell
Dipartemento d'Estructura i Constituents de la Materia, Facultat de Fisica, Universitat de Barcelona, E-08028 Barcelona, Spain
\end{abstract}

(Received 20 August 1991; accepted for publication 25 September 1991)

\begin{abstract}
It is shown that propagation around a circular bend in a quantum wire is well approximated by a one-dimensional problem with a square-well potential replacing the bend. Simple analytic expressions are obtained for the transmission and bound states.
\end{abstract}

Lent ${ }^{1}$ and also Sols and Macucci $^{2}$ have carried out numerical calculations for transmission and bound states of a quantum wire with a circular bend. We shall show here that their results can be understood in detail, and very simply, in the approximation introduced by Jensen and Koppe $^{3}$ that the width of the wire is small compared to its radius of curvature. As we shall see, the results are excellent even when this condition is seriously violated.

Let the circular bend have inner radius $R$ and width $d$, and subtend an angle $2 \Theta$ from the center of curvature. The bend is connected to entrance/exit leads which are straight, and of the same width $d$, as sketched in the inset to Fig. 1. In the straight section we use Cartesian coordinates $(x, r)$, and write the Schrödinger equation

$$
\left(\nabla^{2}+k^{2}\right) \Psi(x, r)=0,
$$

with $k^{2}=2 m^{*} E / \hbar^{2}$. The solutions are a linear combination of channel wave functions

$$
\Psi_{n}(x, r)=e^{ \pm i p_{n} x} \sin [(n \pi / d)(r-R)],
$$

where $(n \pi / d)^{2}+p_{n}^{2}=k^{2}$.

In the bend we use plane polar coordinates $r, \theta$, giving

$$
\frac{\partial^{2} \Psi}{\partial r^{2}}+\frac{1}{r} \frac{\partial \Psi}{\partial r}+\frac{1}{r^{2}} \frac{\partial^{2} \Psi}{\partial \theta^{2}}+k^{2} \Psi=0
$$

This has solutions

$$
\Psi(r, \theta)=Z_{v}(k r) e^{ \pm i v \theta},
$$

where

$$
Z_{v}(k r)=J_{v}(k r) N_{v}(k R)-J_{v}(k R) v_{N}(k r)
$$

vanishes at $r=R$. Then $v$ and $k$ are related by the remaining radial boundary condition

$$
Z_{v}[k(R+d)]=0
$$

and the mode matching at the ends of the circular section. Following Jensen and Koppe ${ }^{3}$ we make the transformation

$$
\Psi(r, \theta)=(1 / \sqrt{r}) \chi(r, \theta)
$$

which gives

$$
\frac{\partial^{2} \chi}{\partial r^{2}}+\frac{1}{4 r^{2}} \chi+\frac{1}{r^{2}} \frac{\partial^{2} \chi}{\partial \theta^{2}}+k^{2} \chi=0
$$

The crucial approximation is to replace $r^{2}$ by an average value $\bar{R}^{2}$ in the denominator. Because Jensen and Koppe were interested only in the limit of vanishing $d$, they simply used $R^{2}$, but it is better to use

$$
\frac{1}{\bar{R}^{2}} \equiv \frac{1}{d} \int_{R}^{R+d} \frac{1}{r^{2}} d r=\frac{1}{R(R+d)}
$$

With this replacement, Eq. (7) becomes

$$
\frac{\partial^{2} \chi}{\partial r^{2}}+\frac{1}{4 \bar{R}^{2}} \chi+\frac{\partial^{2} \chi}{\partial x^{2}}+k^{2} \chi=0
$$

(where $x=\bar{R} \theta$ ), and has separable solutions

$$
\Psi_{n}(x, r)=(1 / \sqrt{r}) \Phi_{n}(x) \sin [(n \pi / d)(r-R)] .
$$

The longitudinal motion in the bend is then the solution of

$$
\frac{\partial^{2} \Phi_{n}}{\partial x^{2}}+\left(p_{n}^{2}+\frac{1}{4 \bar{R}^{2}}\right) \Phi_{n}=0,-a<x<a,
$$

where $a=\bar{R} \Theta$. This is simply motion in a one-dimensional square-well potential of depth

$$
V_{0}=\left(\hbar^{2} / 2 m^{*}\right)(1 / 2 \bar{R})^{2}
$$

and width $2 a=2 \bar{R} \Theta$; with channel energy $p_{n}^{2}=k^{2}$ - $(n \pi / d)^{2}$. Effectively, the approximation replaces the motion in the circular bend by motion in a straight section with this additional attractive potential.

The second approximation one must make is to treat the square root of $r$ in Eq. (10) as constant. Then the various channel wave functions decouple, and the problem becomes strictly one dimensional. This is reasonable providing we remain below the threshold for the second channel; we come back to this point later. In their paper, Jensen and Koppe considered only a complete circle closed on itself, so they could avoid this matching problem. The same is true if one considers a closed circular bend with vanishing boundary condition on the four walls. In either case, the exact solution using the asymptotic form of the Bessel function ${ }^{4}$ verifies the choice of $\bar{R}$ expressed in Eq. (8).

First we apply the approximation to the bound state: from Eq. (11) we write

$$
\Phi(x)=\cos (v \theta)=\cos (v x / \bar{R}),
$$




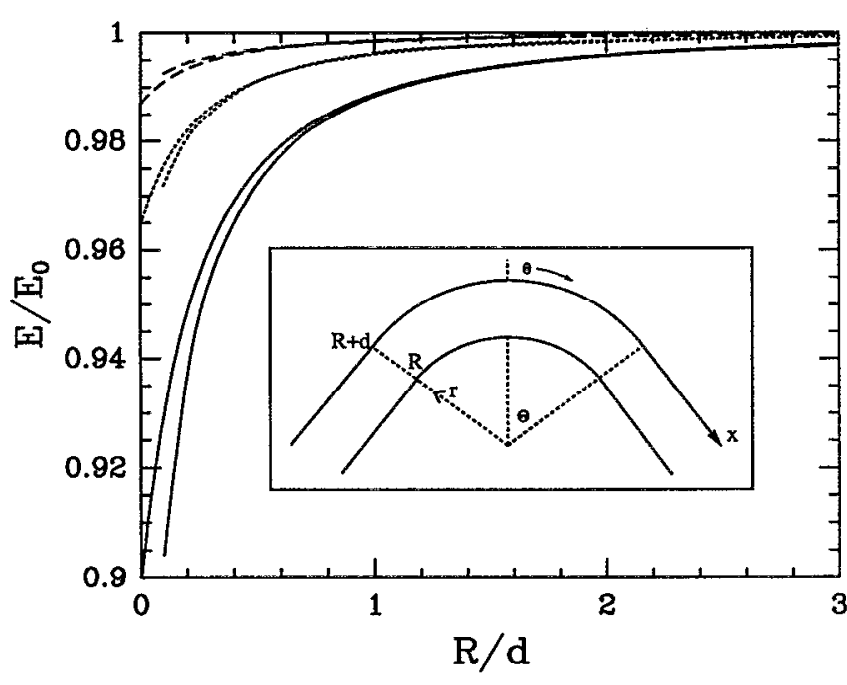

FIG. 1. Bound-state energy as a function of ratio $R / d$ for right-angle (dotted line) and $180^{\circ}$ (dashed line) bends. The lines that terminate before $R=0$ are the result of Sols and Macucci, the others are our Eq. (19). The solid line corresponds to setting $v=0$ in Eq. (19); it is the depth of the square-well potential replacing the bend.

where $v$ will be determined by matching at the boundaries to the leads, in which

$$
\Psi(x, y)=C \sin (\pi y / d) e^{-\kappa|x|} .
$$

Here, $y=r-R$, and $x$ follows continuously from $\bar{R} \theta$ in the bend. In the approximation that the $1 / \sqrt{r}$ factor belonging to $\chi$ is a constant, the matching condition is simply

$$
(v / \bar{R}) \tan v \Theta=\kappa,
$$

where $\kappa \equiv-i p_{1}$. From Eq. (11) we have the binding energy

$$
\kappa^{2}=\left(1 / \bar{R}^{2}\right)\left(\frac{1}{4}-v^{2}\right) .
$$

Combining these gives

$$
\Theta=(1 / v) \tan ^{-1}\left[\sqrt{\left(1 / 4 v^{2}\right)-1}\right] .
$$

Given the bend angle, one can solve for $v$. In the case of a right-angle bend, $\Theta=\pi / 4$, we find $v=0.467$, while for a $180^{\circ}$ bend, $\Theta=\pi / 2, v=0.403$. Introducing the minimum energy for propagation in the straight channels,

$$
E_{0}=\left(\hbar^{2} / 2 m^{*}\right)(\pi / d)^{2},
$$

our result for the energy of the bound state is

$$
E / E_{0}=1-(d / 2 \pi \bar{R})^{2}\left(1-4 v^{2}\right) .
$$

Recall that in Eq. (8) we chose $\bar{R}^{2}=R(R+d)$. This will predict a diverging binding energy for small $R$. However, on dimensional grounds, the energy can depend only on $d^{2}$ in this limit. This suggests the replacement of Eq. (8) by

$$
1 / \overline{R^{2}}=\frac{1}{(R+d / 2)^{2}},
$$

which uses the arithmetic rather than the geometric mean of the two radii of the bend. We have replaced the circular bend by an equivalent rectangle. The choice $\bar{R}=R+d / 2$ ensures that in this replacement the area and the perimeter of the bend are preserved, and also the sum of the four angles at the corners of the figure. (This would not be so for the geometric mean.) It has been shown by Balian and Bloch ${ }^{5}$ that the spectral density can be expressed by an asymptotic expansion in terms of the area, perimeter, curvature, etc. of the figure, so the choice of the arithmetic mean should be very accurate, as indeed we find it to be.

In Fig. 1 we compare the result of our analytic approximation with the calculation of Sols and Macucci. [The solid line corresponds to taking $v=0$ in Eq. (19).] We find excellent agreement with their curves for both the rightangle and the $180^{\circ}$ bends except at the smallest values of $R / d$. Since the approximation is based on considering $d / R$ to be small, this minor disagreement is hardly surprising.

To compute the transmission coefficient, one takes plane waves for the longitudinal propagation both in the bend and in the leads. From Eqs. (1) and (11) one has

$$
p^{2}=q^{2}-(1 / 2 \bar{R})^{2}=\left(2 m^{*} E / \hbar^{2}\right)-(\pi / d)^{2},
$$

where $p$ and $q$ are, respectively, the wave numbers in the leads and in the bend. As remarked before the problem is equivalent to that of transmission in a one-dimensional square-well potential. One can therefore write the transmission probability as ${ }^{6}$

$$
T^{-1}=1+\left(q^{2}-p^{2}\right)^{2} \sin ^{2}(2 q a) / 4 p^{2} q^{2} .
$$

In order to compare with the calculations of Lent, ${ }^{1}$ we put the momenta into the dimensionless form $P=p d / \pi$. The variable against which Lent plots $T$ is actually $k d / \pi$ $=\sqrt{1+P^{2}}$. We find

$$
T^{-1}=1+\lambda^{4} \sin ^{2}(2 q a) / 4 P^{2}\left(P^{2}+\lambda^{2}\right),
$$

where

$$
\lambda=d / 2 \pi \bar{R}
$$

and

$$
2 q \dot{a}=(\Theta / \lambda) \sqrt{P^{2}+\lambda^{2}} .
$$

Our results are shown in Fig. 2 for the cases computed by Lent. It can be seen that the agreement is excellent, confirming that this simple analytical approximation can be reliably used for a wide range of $R / d$ ratios. Furthermore it has the advantage of being equally easy to use for any bend angle.

Until now only transmission in the lowest mode has been discussed. For all energies above threshold other transverse modes contribute to transmission. In their Fig. 2, Sols and Macucci show the partial transmission probabilities into various channels. If one sums these, the total transmission probability is almost unity at all energies above threshold, from each entrance channel. In the model as presented above, the bent wire has been reduced to a strictly one-dimensional problem. A plot of the conductance will be of the familiar step form, with each step constructed by displacing the $n=1$ conductance curve and adding it on top of the previous steps. ${ }^{7}$ 


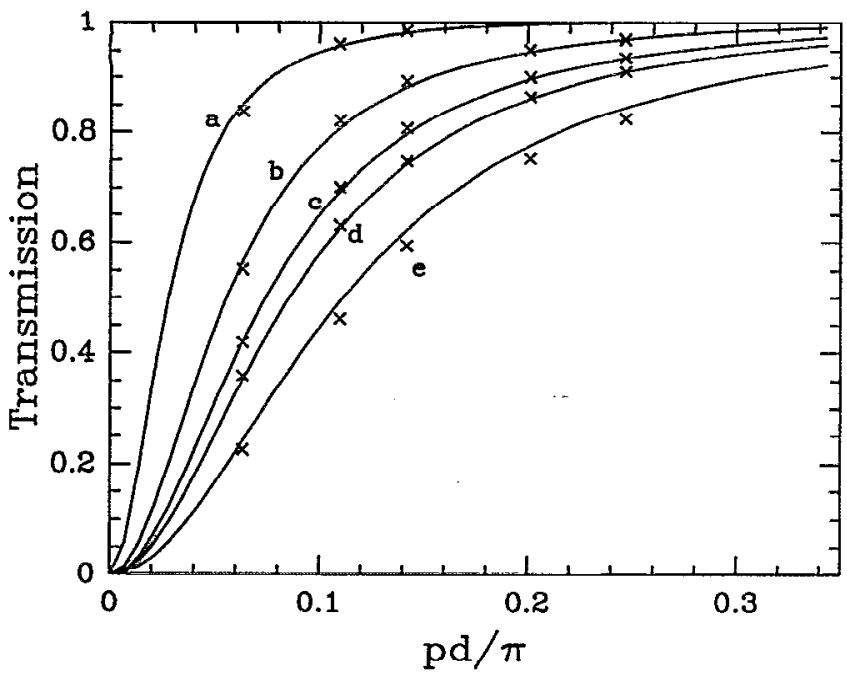

FIG. 2. Transmission probability near threshold for a right-angle bend as function of the dimensionless momentum $P=p d / \pi$, using Eqs. (23)(25). For comparison with Lent, the lines correspond to values of $\bar{R} / d$ as follows: (a) 2.0 , (b) 1.0 , (c) 0.75 , (d) 0.65 , and (e) 0.5 . The crosses mark points read from his graph.

Due to the channel decoupling, in our approximation we predict a bound state for each transverse mode $n$. A trivial extension of the derivation leading to Eq. (19) shows that the energy shift $\Delta E_{n}$ with respect to the threshold energy for propagation in the channel is independent of $n$ and has the same form as in Eq. (19):

$$
\Delta E_{n} / E_{0}=-(d / 2 \pi \bar{R})^{2}\left(1-4 v^{2}\right) .
$$

When coupling is restored these states become quasibound and appear as subthreshold resonances in the transmission probabilities computed in Refs. 1 and 2. For a $90^{\circ}$ bend and a ratio $\bar{R} / d=0.7$, Sols and Macucci find the first of these at $\Delta E_{1} / E_{0} \approx 0.006$ and state that the second appears at a similar distance below its threshold. With the above equation we predict $\Delta E_{n} / E_{0}=0.0066$ for both cases, again in good agreement with their results and therefore confirming the physical interpretation of these resonances.

Lent has also considered a case where the confining walls of the channels are not infinite, but still very high at
$48 E_{0}$. Above the second channel threshold he found surprisingly large effects on the reflectivity from this modest change in confinement (see his Fig. 3). Since in a real device the confining walls are certainly not infinite, his result implies that the hard-wall models generally employed are not sufficient to obtain a detailed picture of the transmission above the second channel threshold. In this situation, the one-dimensional model employed here is perfectly adequate, as it duplicates the exact results in the region where they are physically meaningful.

In conclusion, we have extended the approximation of Jensen and Koppe to include terms of order $(d / R)^{2}$. This replaces the propagation around a circular bend by propagation in a straight section with an attractive square potential. This approximation gives excellent agreement with the numerical calculations of Lent and Sols and Macucci. The remaining error in our results is due to the neglect of mode mixing. In a previous paper ${ }^{8}$ we showed that the bound states in a rectangular bend could be similarly approximated by a one-dimensional problem with an attractive potential. Thus the main features of bent quantum wires can be understood from the simple formulas given in Eqs. (19)-(20) and (22)-(25). The parametric dependence-of the characteristics of the device on its geometry is obvious from these formulas.

We are grateful to NSERC Canada for continued support under operating Grant No. A-3198 (D.W.L.S. and H.W.). The work of J.M. is partially supported under Grant No. PB-87-0311 from DGICYT Spain. In addition, D.W.L.S. is grateful to the Spanish Ministry of Science and Education (DGICYT) for support as a visiting professor in Barcelona where part of this work was done.

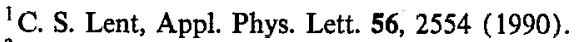

${ }^{2}$ F. Sols and M. Macucci, Phys. Rev. B 41, 11887 (1990).

${ }^{3}$ H. Jensen and H. Koppe, Ann. Phys. 63, 586 (1971).

${ }^{4} \mathrm{M}$. Goldstein and R. M. Thaler, Mathematical Tables and other Aids to Computation 12, 18 (1958).

${ }^{5}$ R. Balian and C. Bloch, Ann. Phys. 60, 401 (1970); 64, 271 (1971); 69, 76 (1972).

${ }^{6}$ E.' Merzbacher, Quantum Mechanics, 2nd ed. (Wiley, New York, 1970), p. 109.

${ }^{7}$ IIua Wu, D. W. L: Sprung, J. Martorell, and S. Klarsfeld, Phys. Rev. B 44, 6351 (1991).

${ }^{8}$ Hua Wu, D. W. L. Sprung, J. Martorell, and S. Klarsfeld (unpublished). 